\title{
Retrospective Study on the Microbial Drug Resistance Profile and Predictive Risk Factors in Early Infection of Open Fractures
}

Tai KMa, Sulong $A F^{a}$, Mohd. Yusof $N^{a}$

${ }^{a}$ Department of Orthopedic, Traumatology and Rehabilitation, International Islamic University of Malaysia

\section{ABSTRACT}

Introduction: This study was conducted to evaluate the susceptibility of microorganisms involved in early infection of open fractures in orthopaedics to current prophylactic antibiotics practice and determine the association of various parameters related to the causative organism, patient, injury and management to the resistance of such microorganisms. Methods: This is a retrospective study that reviewed cases collected from the surgical operations record from January 2015 to December 2017 in Hospital Tengku Ampuan Afzan, Kuantan. A total of 179 cases were collected with 110 cases fulfilling the proposed inclusion / exclusion criteria. They all underwent routine debridement followed by culture and sensitivity. Previous clinical records were traced to identify the various risk factors for infection and evaluate these against the resistance of the microorganisms to prophylactic antimicrobial drugs. Results: Admission to the intensive care unit, blood transfusion, antibiotic regime and duration, fracture grade, injury severity score and duration of admission were all significantly associated with resistance of microorganism. The study also demonstrated the predominance of Gram-negative microorganisms constituting $65.9 \%$ of isolates. Gramnegative microorganisms were seen more in higher fracture grades and Injury Severity Scale, and longer duration of admission and antibiotic treatment. The overall susceptibility of microorganisms to antibiotics was also demonstrated with up to $68.6 \%$ resistance to cefuroxime and $36.9 \%$ to Gentamicin. Staphylococcus aureus and MRSA were the commonest Gram-positive organisms while Pseudomonas and Acinetobacter spp. the commonest Gram-negatives. Conclusion: Admission to Intensive Care Unit, blood transfusion, antibiotic regime and duration, fracture grade, injury severity score and duration of antibiotics have a significant positive predictive value to the development of resistance of microorganisms. The shift to predominantly Gram-negative microorganisms indicate an urgency to modify management of open fracture to achieve optimal results. This will also prevent the emergence of new multi-drug resistance microorganism which is already a worldwide public health problem.

KEYWORDS: infected open fracture, microorganism and antibiotic resistance

\section{INTRODUCTION}

Open fracture is generally defined as break in structural continuity of bone exposed to external surrounding. The incidence of open fracture ranges from 11.1 to 21.3 per 100000 per year. ${ }^{1,2}$ Road traffic accidents are very common in Malaysia with an official record of a total 477204 accidents and

Corresponding author:

Dr. Tai Ken Meng

Department of Orthopedic,

Traumatology and Rehabilitation, International Islamic University Malaysia, Jalan Sultan Ahmad Shah, 25200 Kuantan, Pahang, Malaysia.

Tel No: +609-5912500

Email: kenmeng85@hotmail.com
6915 deaths in $2013 .^{3}$ Injuries due to road traffic accidents ranked third (8.2\%) after pregnancy and respiratory disorders as a cause of hospitalization in Malaysia. ${ }^{4}$

With such numbers, the management of open fracture is challenging for orthopaedic surgeons due to the significant risk of complications especially infection. Despite the advancement of medical facilities and antibiotic prophylaxis, the reported frequencies of open fractures were $0-8.5 \%$ for grade 1 fractures, $9.4-17.6 \%$ for grade II, $21.1-41.2 \%$ for grade IIIA and $41.2-44 \%$ for grade IIIB and IIIC. ${ }^{5,6}$ This study primarily aims to determine the susceptibility of causative microorganisms to prophylactic 
antibiotics used in practice. The other objective is to evaluate the association of various risk factors such as age, underlying comorbidities, mechanism of injury, site of injury, injury severity score (ISS), duration of admission, duration of antibiotics and its regime, intensive care unit (ICU) admission and blood transfusion to the development of resistant microorganisms.

\section{MATERIALS AND METHODS}

This is a retrospective cross-sectional study involving patients treated for early infection of open fractures in HTAA over a 2 year period from 1 January 2015 to 31 December 2017. During the sample collection, diagnosis of early infection in open fracture is made based on patients' clinical signs and symptoms. Definition of infection in this study is a presentation of local swelling, erythema or tenderness which requires surgical debridement within 6 months from the time of initial open fracture.

Further data and radiographs were obtained from available medical records. Only patients subjected to standard treatment protocol for open fractures which includes prophylactic antibiotics, wound debridement and skeletal stabilization were included. Patients with pre-existing infection, receiving antibiotics prior to admission and those who refused antibiotics or wound debridement were excluded from the study. This study was approved by Medical Research and Ethics Committee (MREC) and International Islamic University (IIUM) Research Ethics Committee.

Following diagnosis, patients underwent routine irrigation, wound debridement and antibiotic administration, which is based on local guidelines which include intravenous Cefuroxime $1.5 \mathrm{gm}$ as loading followed by $750 \mathrm{mg} 8$ hourly for fracture grade I and II with the addition of intravenous Gentamicin $5 \mathrm{mg} / \mathrm{kg}$ every 24 hours and intravenous Metronidazole $500 \mathrm{mg} 8$ hourly for fracture grade III. Culture and sensitivity obtained during debridement were monitored to determine the microorganism and their sensitivity profile. Microorganisms were divided into 2 categories based on their sensitivity to prophylactic antibiotics.

Univariate analysis for categorical variables was applied to determine the association of multiple variables with the susceptibility profile of bacterial isolates to prophylactically given antimicrobial drugs. The Pearson chi-square was done using IBM SPSS version 23 and Fisher's exact test using STATA 11.0 .

\section{RESULTS}

A total of 179 cases were in the hospital record having undergone wound debridement for infected, open fracture from $1^{\text {st }}$ January 2015 to $31^{\text {st }}$ December 2017. A total of 110 cases fulfilled the inclusion and exclusion criteria. There were 90 $(81.8 \%)$ males and $20(18.2 \%)$ females. Patients with age ranging from 21-40 years were more likely to incur such injuries counting 42 cases (38.2\%). The majority of the patients $75.5 \%(83 / 110)$ did not have underlying medical conditions and 84 cases (76.4\%) were involved in road traffic accidents. Lower limb involvement was seen in 91 cases $(82.7 \%)$ with the tibia being the most frequently involved bone $41.8 \%$ ( $46 / 110$ cases).

Gustilo Anderson open fracture classification was used to determine fracture grade. Grade IIIA comprised $45.5 \%$ (50/110) of cases, Grade IIIB and II were equally represented with 25 cases $(22.7 \%)$, Grade IIIC with 9 cases $(8.2 \%)$ ad Grade I with 1 case $(0.9 \%)$. Most of the cases had ISS score of moderate with 42 cases (38.2\%). 24 cases $(21.8 \%)$ required ICU admission, 86 cases $(78.2 \%)$ were hospitalized for more than 10 days while the majority of the 66 cases $(60.0 \%)$ did not require blood transfusion. Most of the cases $(70 \%)$ underwent debridement within 12 to 24 hours. Combination of 3 prophylactic antibiotics was given to 57 cases $(51.8 \%)$ and duration of antibiotic treatment of more than 10 days was encountered in 85 cases (77.3\%).

168 microorganisms were cultured from the 110 cases. Most of the cases $61.8 \%(68 / 110)$ had a single bacterial isolate. In 41 cases (37.3\%) the bacterial isolates were sensitive to the prophylactic antibiotics used, while 55 cases (50\%) were infected with resistant organisms. Meanwhile sensitivity was not done in 14 cases due to polymicrobial growth. Gram-negative microorganisms were encountered in 91 cases $(65.9 \%)$ while 37 cases $(26.8 \%)$ had gram-positive organisms and mixed culture were detected in 10 cases (7.3\%). Pseudomonas species were involved in 30 cases $(24 \%)$ followed by Staphylococcus aureus in 19 cases (15.2\%), and 
Methicillin-resistant Staphylococcus aureus and Acinetobacter species in 17 cases each (13.6\%). Enterobacter species and Klebsiella pneumonia contributed 12 cases each $(9.6 \%)$, and E. coli 7 cases (5.6\%). Polymicrobial infection with 3 microorganisms was observed in 11 cases (8.8\%) while other microorganisms were involved in less than $5 \%$ of cases.

Gender, age category, underlying medical conditions, side of injury and duration from injury to debridement were not related to resistance of microorganisms. As seen in Table 1 and 2, fracture grade, ISS score, ICU admission and blood transfusion are all significantly associated with the resistance of microorganisms ( $p$ value $<0.01$ ). Prophylactic antibiotic regime, duration of antibiotics and admission also revealed a significant statistical association ( $p$ value $<0.01$ )

Antibiotic susceptibility was done for both Grampositive and Gram-negative microorganism. In Staphylococcus aureus group, there was resistance to Penicillin G in all 5 cases tested (100\%), while resistance to Ampicillin was observed in 9 cases (69.2\%) (Table 3). In MRSA group, however all 17 cases $(100 \%)$ were sensitive to Fusidic acid, 13 cases (100\%) to Rifampicin, 7 cases $(100 \%)$ to Vancomycin and 14 cases (82.4\%) to Gentamicin. With regards to Gram-negative microorganism, Acinetobacter species was demonstrated to be resistant in 17 cases (100\%) to Cefuroxime, Amikacin, Piperacilin, Ceftazidime and Tazobactam (Table 4$)$. It is also resistant in 16 cases $(100 \%)$ tested to Imipenem and Meropenem while resistant in 16 cases (94.1\%) to Gentamicin and 14 cases (93.3\%) to Ciprofloxacin. All Acinetobacter infected cases were sensitive to Colistin group antibiotics with 17 cases $(100 \%)$. Pseudomonas group reported 18 cases $(62.1 \%)$ resistance to Cefuroxime and 17 cases $(58.6 \%)$ to Ceftazidime. 22 cases $(78.6 \%)$ however, show susceptibility to Gentamicin, 18 cases $(66.7 \%)$ to Tazobactam and 20 cases $(69 \%)$ to Ciprofloxacin. There was 1 case of ESBL producing pseudomonas which was resistant to Cefuroxime, Ceftazidime and Gentamicin but sensitive to Imipenem and Meropenem.

In $E$. coli group, there were 3 cases infected with ESBL producer $E$. coli, all resistant to Ceftazidime. Enterobacter aerogenes group shows $2 / 6$ cases (33\%) with resistance to Cefuroxime and
Ceftazidime but $6 / 6$ cases $(100 \%)$ sensitive to Gentamicin.

Table 1: Association between Various Variables with the Antimicrobial Susceptibility Profile of the Isolated Microorganisms

\begin{tabular}{lllll}
\hline \multirow{2}{*}{ Characteristics } & \multicolumn{2}{l}{ Sensitivity, } & $\begin{array}{l}x^{2} \text { - } \\
\text { Statis- } \\
\text { tics (df) }\end{array}$ & $p$-value \\
\cline { 2 - 4 } & No & Yes & & \\
\hline Gender & & & & \\
Male & $41(52.6)$ & $37(47.4)$ & & \\
Female & $14(77.8)$ & $4(22.2)$ & & \\
$\begin{array}{l}\text { Age category } \\
\text { (Years) }\end{array}$ & & & & \\
$<20$ & $17(56.7)$ & $13(43.3)$ & & \\
$21-40$ & $21(65.6)$ & $11(34.4)$ & & \\
$41-59$ & $7(36.8)$ & $12(63.2)$ & $4.70(3)$ & 0.195 \\
$>60$ & $10(66.7)$ & $5(33.3)$ & &
\end{tabular}

Medical Illness

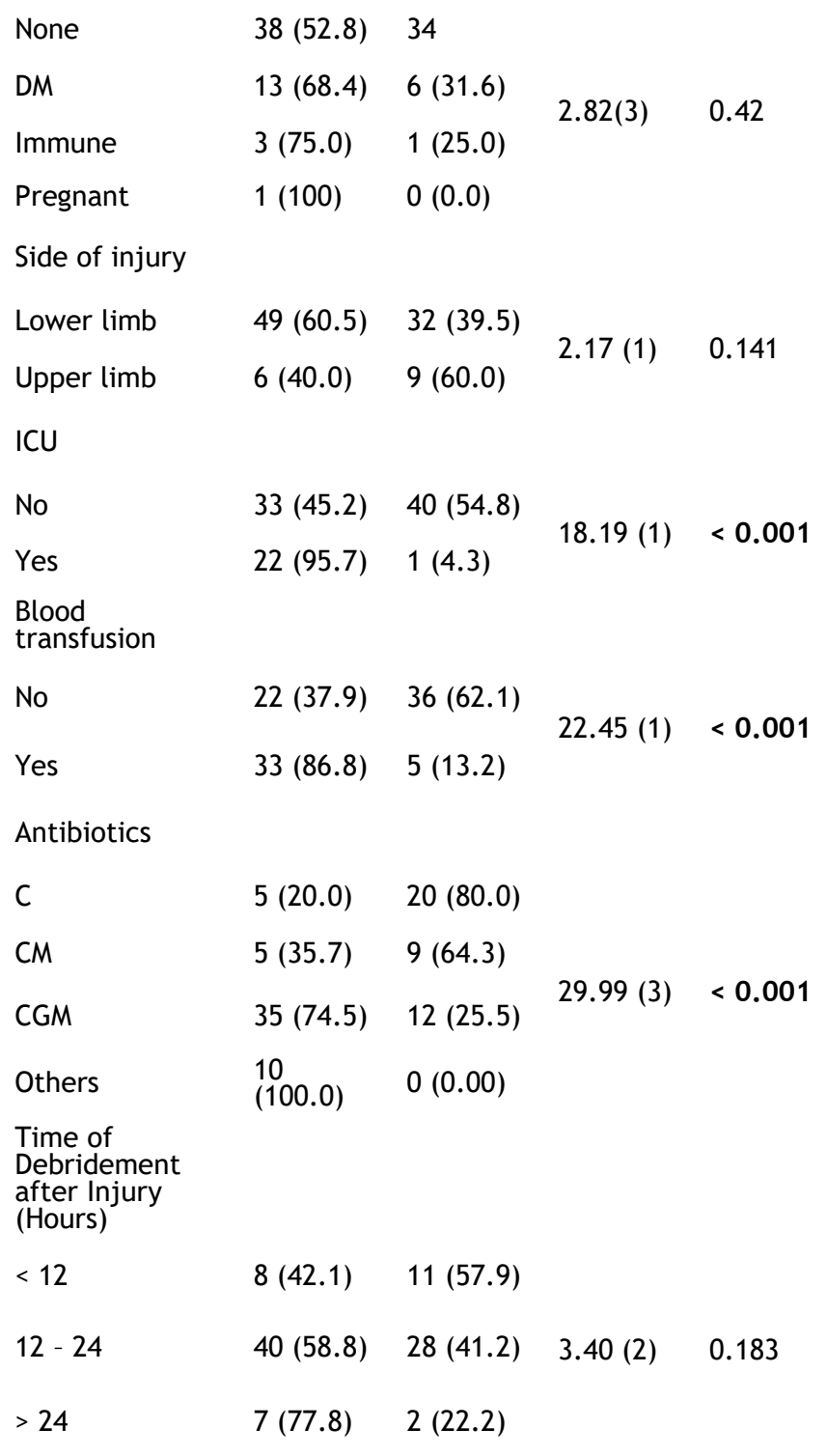


Table 2: Association between Various Variables with the Antimicrobial Susceptibility Profile of Isolated Microorganisms

\begin{tabular}{|c|c|c|c|c|}
\hline \multirow{2}{*}{ Characteristics } & \multicolumn{2}{|c|}{$\begin{array}{l}\text { Sensitivity, } \\
\mathrm{n}(\%)\end{array}$} & \multirow{4}{*}{$\begin{array}{l}\text { X2 - } \\
\text { statistics } \\
\text { (df) }\end{array}$} & \multirow[t]{2}{*}{$p$-value } \\
\hline & \multicolumn{2}{|c|}{ Grade } & & \\
\hline I & $0(0.0)$ & $\begin{array}{l}1 \\
(100.0)\end{array}$ & & \\
\hline II & $3(13.0)$ & $\begin{array}{l}20 \\
(87.0)\end{array}$ & & \\
\hline IIIA & $\begin{array}{l}23 \\
(53.5)\end{array}$ & $\begin{array}{l}20 \\
(46.5)\end{array}$ & $41.6(4)$ & $<0.001^{\mathrm{b}}$ \\
\hline IIIB & $\begin{array}{l}22 \\
(100.0)\end{array}$ & $0(0.0)$ & & \\
\hline IIIC & $\begin{array}{l}7 \\
(100.0)\end{array}$ & $\begin{array}{l}0 \\
(0.0)\end{array}$ & & \\
\hline \multicolumn{5}{|l|}{$\begin{array}{l}\text { Injury Severity } \\
\text { Scale (ISS) }\end{array}$} \\
\hline Minor & $\begin{array}{l}6 \\
(19.4)\end{array}$ & $\begin{array}{l}25 \\
(80.7)\end{array}$ & & \\
\hline Moderate & $\begin{array}{l}23 \\
(63.9)\end{array}$ & $\begin{array}{l}13 \\
(36.1)\end{array}$ & & \\
\hline Serious & $8(88.9)$ & $\begin{array}{l}1 \\
(11.1)\end{array}$ & $31.44(4)$ & $<0.001^{b}$ \\
\hline Severe & $\begin{array}{l}15 \\
(88.2)\end{array}$ & $\begin{array}{l}2 \\
(11.8)\end{array}$ & & \\
\hline Critical & $\begin{array}{l}3 \\
(100.0)\end{array}$ & $\begin{array}{l}0 \\
(0.0)\end{array}$ & & \\
\hline Maximum & $\begin{array}{l}0 \\
(0.0)\end{array}$ & $\begin{array}{l}0 \\
(0.0)\end{array}$ & & \\
\hline \multicolumn{5}{|l|}{$\begin{array}{l}\text { Mechanism of } \\
\text { Injury }\end{array}$} \\
\hline RTA & $\begin{array}{l}49 \\
(67.1)\end{array}$ & $\begin{array}{l}24 \\
(32.9)\end{array}$ & & \\
\hline Industrial & $\begin{array}{l}4 \\
(30.8)\end{array}$ & $\begin{array}{l}9 \\
(69.2)\end{array}$ & & \\
\hline Domestic & $\begin{array}{l}2 \\
(33.3)\end{array}$ & $\begin{array}{l}4 \\
(66.7)\end{array}$ & $13.40(4)$ & $0.003^{b}$ \\
\hline Sports & $\begin{array}{l}0 \\
(0.0)\end{array}$ & $\begin{array}{l}1 \\
(100.0)\end{array}$ & & \\
\hline Farm & $\begin{array}{l}0 \\
(0.0)\end{array}$ & $\begin{array}{l}3 \\
(100.0)\end{array}$ & & \\
\hline \multicolumn{5}{|l|}{$\begin{array}{l}\text { Duration of } \\
\text { admission } \\
\text { (Days) }\end{array}$} \\
\hline$<3$ & $\begin{array}{l}0 \\
(0.0)\end{array}$ & $\begin{array}{l}0 \\
(0.0)\end{array}$ & & \\
\hline $3-6$ & $\begin{array}{l}2 \\
(10.5)\end{array}$ & $\begin{array}{l}17 \\
(89.5)\end{array}$ & & \\
\hline $7-10$ & $\begin{array}{l}2 \\
(66.7)\end{array}$ & $\begin{array}{l}1 \\
(33.3)\end{array}$ & $21.18(2)$ & $<0.001^{b}$ \\
\hline$>10$ & $\begin{array}{l}51 \\
(68.9)\end{array}$ & $\begin{array}{l}23 \\
(31.1)\end{array}$ & & \\
\hline \multicolumn{5}{|l|}{$\begin{array}{l}\text { Duration of } \\
\text { antibiotics } \\
\text { treatment } \\
\text { (Days) }\end{array}$} \\
\hline$<3$ & $\begin{array}{l}0 \\
(0.0)\end{array}$ & $\begin{array}{l}0 \\
(0.0)\end{array}$ & & \\
\hline $3-5$ & $\begin{array}{l}2 \\
(14.3)\end{array}$ & $\begin{array}{l}12 \\
(85.7)\end{array}$ & & \\
\hline 6- 7 & $\begin{array}{l}0 \\
(0.0)\end{array}$ & $\begin{array}{l}6 \\
(100.0)\end{array}$ & $23.45(3)$ & $<0.001^{\mathrm{b}}$ \\
\hline 8- 10 & $\begin{array}{l}2 \\
(66.7)\end{array}$ & $\begin{array}{l}1 \\
(33.3)\end{array}$ & & \\
\hline$>10$ & $\begin{array}{l}51 \\
(69.9)\end{array}$ & $\begin{array}{l}22 \\
(42.7)\end{array}$ & & \\
\hline
\end{tabular}

Pearson chi-square test was applied.

' Fisher's exact test was applied; Assumption of Pearson chi-square test was violated: expected count of less than 5 was $>20 \%$.
With regards to Enterobacter gergoviae, $3 / 4$ cases (75\%) were resistant to Cefuroxime but $4 / 4$ cases (100\%) were sensitive to Gentamicin. Proteus species showed good sensitivity to Cefuroxime and Gentamicin with 4 cases (100\%) susceptible to them. Klebsiella pneumoniae was demonstrated with 4 cases $(50 \%)$ sensitive to Cefuroxime and 6 cases (75\%) to Gentamicin. There were 2 cases of ESBL producing Klebsiella pneumoniae both resistant to Cefuroxime, Ceftazidime and Gentamicin but sensitive to Imipenem and Meropenem. There were also 2 reported cases of Carbapenem-resistant (CRE) Klebsiella pneumoniae, which were also resistant to all antibiotics tested except Colistin to which they were both sensitive.

This study illustrates the susceptibility of both Gram -positive and negative microorganisms to the antibiotics tested. With regards to Gram-positive microorganisms, $91.4 \%(32 / 35)$ were sensitive to Fusidic acid, $91.9 \%(34 / 37)$ to Gentamicin, $100 \%$ to Rifampicin (13/13) and 100\% (7/7) to Vancomycin. Resistance to Clindamycin was found in $9 / 14$ cases (64.3\%), Erythromycin in $17 / 36$ cases (47.2\%), Oxacillin in $22 / 36$ cases $(61.1 \%$ ) and Ampicillin in $14 / 18$ cases $(77.8 \%)$. Gram-negative microorganisms demonstrated resistance to Cefuroxime in $59 / 86$ cases $(68.6 \%)$, Gentamicin in $31 / 84$ cases $(36.9 \%)$, Piperacillin in $21 / 23$ cases (91.3\%), Ceftazidime in $57 / 75$ cases $(76.0 \%$ ), Carbapenem group in $27 / 41$ cases (65.9\%) and Ampicillin in 35/37 cases (94.6\%) while sensitive to Colistin in $27 / 27$ cases $(100 \%)$.

\section{DISCUSSION}

Infection in open fracture has always been a dreaded complication in the field of orthopaedics. Despite the magnitude of advancement in the past years to curb this problem, infection rate seems to be on the rise and more worrisomely is the rise of resistant microorganisms. Higher percentage of infected open fractures was noted among males in this study, which is although concordant with findings of other studies ${ }^{7}$ but was not statistically significant. In terms of age, this study reported no significant association with resistance of microorganisms despite the common knowledge of higher incidence of patients suffer from other medical illnesses in the older age group. This is similar to a study showing the association between antibiotic resistance and community-acquired bloodstream infection. ${ }^{8}$ 
Table 3: Antibiogram of the Gram-positive Bacterial Isolates

\begin{tabular}{|c|c|c|c|c|c|c|c|c|c|c|c|}
\hline \multirow[b]{2}{*}{$\begin{array}{l}\text { Micro- } \\
\text { organism }\end{array}$} & \multirow[b]{2}{*}{$\begin{array}{l}\mathrm{RX} \\
\mathrm{N}\end{array}$} & \multicolumn{10}{|c|}{ Susceptibility to antimicrobial agents no. (\%) } \\
\hline & & $\begin{array}{l}\mathrm{FA} \\
\text { no. }= \\
35\end{array}$ & $\begin{array}{l}\text { no. }=37 \\
\text { no }\end{array}$ & $\begin{array}{l}\text { TS } \\
\text { no. }=35\end{array}$ & $\begin{array}{l}\mathrm{R} \\
\text { no. }= \\
13\end{array}$ & $\begin{array}{l}V \\
\text { no. }= \\
7\end{array}$ & $\begin{array}{l}\text { C } \\
\text { no. }=\end{array}$ & $\begin{array}{l}\text { E } \\
\text { no. }= \\
36\end{array}$ & $\begin{array}{l}P \\
\text { no. }= \\
5\end{array}$ & $\begin{array}{l}0 \\
\text { no. }= \\
36\end{array}$ & $\begin{array}{l}\text { A } \\
\text { no. }= \\
18\end{array}$ \\
\hline \multirow{2}{*}{$\begin{array}{l}\text { Staph. } \\
\text { aureus }\end{array}$} & $\mathbf{R}$ & $\begin{array}{l}2 \\
(11.8)\end{array}$ & - & $1(5.9)$ & - & - & - & - & $\begin{array}{l}5 \\
(100.0)\end{array}$ & $\begin{array}{l}4 \\
(22.2)\end{array}$ & $9(69.2)$ \\
\hline & S & $\begin{array}{l}15 \\
(88.2)\end{array}$ & $\begin{array}{l}19 \\
(100.0)\end{array}$ & $\begin{array}{l}16 \\
(94.1)\end{array}$ & - & - & - & $\begin{array}{l}18 \\
(100.0)\end{array}$ & - & $\begin{array}{l}14 \\
(77.8)\end{array}$ & $4(30.8)$ \\
\hline \multirow{3}{*}{ MRSA } & $\mathbf{R}$ & - & $3(17.6)$ & $3(17.6)$ & - & - & $\begin{array}{l}9 \\
(64.3)\end{array}$ & $\begin{array}{l}17 \\
(100.0)\end{array}$ & - & $\begin{array}{l}17 \\
(100.0)\end{array}$ & $\begin{array}{l}5 \\
(100.0)\end{array}$ \\
\hline & & & & & & & & & & & \\
\hline & S & $\begin{array}{l}17 \\
(100.0)\end{array}$ & $\begin{array}{l}14 \\
(82.4)\end{array}$ & $\begin{array}{l}14 \\
(82.4)\end{array}$ & $\begin{array}{l}13 \\
(100.0)\end{array}$ & $\begin{array}{l}7 \\
(100.0)\end{array}$ & $\begin{array}{l}5 \\
(35.7)\end{array}$ & - & - & - & - \\
\hline \multirow[b]{2}{*}{ MRCONS } & $\mathbf{R}$ & $\begin{array}{l}1 \\
(100.0)\end{array}$ & - & - & - & - & - & - & - & $\begin{array}{l}1 \\
(100.0)\end{array}$ & - \\
\hline & S & - & $\begin{array}{l}1 \\
(100.0)\end{array}$ & $\begin{array}{l}1 \\
(100.0)\end{array}$ & - & - & - & $\begin{array}{l}1 \\
(100.0)\end{array}$ & - & - & - \\
\hline \multirow[b]{2}{*}{ Total } & $\mathbf{R}$ & $3(8.6)$ & $3(8.1)$ & 4 (11.4) & - & - & $\begin{array}{l}9 \\
(64.3)\end{array}$ & $\begin{array}{l}17 \\
(47.2)\end{array}$ & $\begin{array}{l}5 \\
(100.0)\end{array}$ & $\begin{array}{l}22 \\
(61.1)\end{array}$ & $\begin{array}{l}14 \\
(77.8)\end{array}$ \\
\hline & $S$ & $\begin{array}{l}32 \\
(91.4)\end{array}$ & $\begin{array}{l}34 \\
(91.9)\end{array}$ & $\begin{array}{l}31 \\
(88.6)\end{array}$ & $\begin{array}{l}13 \\
(100.0)\end{array}$ & $\begin{array}{l}7 \\
(100.0)\end{array}$ & $\begin{array}{l}5 \\
(35.7)\end{array}$ & $\begin{array}{l}19 \\
(52.8)\end{array}$ & - & $\begin{array}{l}14 \\
(38.9)\end{array}$ & $4(22.2)$ \\
\hline
\end{tabular}

FA = Fusidic asid; G = Gentamin; TS = Tri-Sulf; R = Rifampicin; V = Vancomycin; $\mathrm{C}=$ Clindamycin; $\mathrm{E}=$ Erythromycin; $\mathrm{P}=$ Penicillin; $\mathrm{O}=$ Oxacillin; $\mathrm{A}$ = Ampicillin

Road traffic accident was involved in majority of cases with $76.4 \%$ in this study which is similar to other studies but reported at a lower rate with $46-69 \% .6,9$ This study however illustrated no statistically significant correlation. There was no statistically significant correlation between presence of underlying medical condition to resistance of microorganisms.

Other studies reported no correlation of diabetes, renal failure or malignancy to resistance of microorganisms. ${ }^{8,10}$ Lower limb involvement is seen in $82.7 \%$ of cases and tibia was the primary bone involved in an infected open fracture with a prevalence of $41.8 \%$. These findings are similar to several other studies but at a lower rate where tibia was involved in $52-75 \%$ of cases. ${ }^{11,12}$ This study did not show any statistically significant association between side of injury to resistance of microorganism.

Gustilo Anderson grade IIIA fractures were the most frequently associated (45.5\%) with early infection of open fracture, while grade III as a whole showed an infection rate of $76 \%$. This finding is similar to that reported by several studies within the same region. ${ }^{5,6}$ This study demonstrates statistically significant association between grades of fracture to resistance of microorganisms with $p$-value of less than 0.001 and significant linear by linear association. Many studies have demonstrated relation between infection rate and fracture grade but not in terms of microorganism or their resistance. ${ }^{9,13}$ A study involving mandibular fracture demonstrated significant association between comminuted fractures with multidrug-resistant bacterial infection. ${ }^{14}$

Majority of cases $38.2 \%$ fell into the moderate ISS with which was significantly associated with resistance in isolated microorganisms ( $p$-value < 0.001 ). Some studies demonstrated association of ISS to infection ${ }^{15}$ while others showed no correlation. ${ }^{12}$ They however did not invoke resistance to antimicrobials. Caricato et al. however described predominance of Acinetobacter baumanii which are multi resistant in trauma patients with higher ISS. ${ }^{16}$ ICU admission was encountered in $21.8 \%$ of cases but 95.7\% of them involved resistant microorganisms which was statistically significant ( $P$ value $<0.001$ ). The need for frequent contact with medical personnel and invasive monitoring jeopardize patient's protective barrier. 
Table 4: Antibiogram of the Gram-negative Bacterial Isolates

\begin{tabular}{|c|c|c|c|c|c|c|c|c|c|c|c|}
\hline \multirow[b]{2}{*}{$\begin{array}{l}\text { Microorganis } \\
\text { m }\end{array}$} & \multirow[b]{2}{*}{ RXN } & \multicolumn{10}{|c|}{ Susceptibility to antimicrobial agents no. (\%) } \\
\hline & & $\begin{array}{l}\mathrm{Ce} \\
\text { no. }=86\end{array}$ & $\begin{array}{l}\text { Co } \\
\text { no. }=27\end{array}$ & $\begin{array}{l}P \\
\text { no. }=23\end{array}$ & $\begin{array}{l}\text { G } \\
\text { no. }= \\
84\end{array}$ & $\begin{array}{l}\text { AS } \\
\text { no. }=35\end{array}$ & $\begin{array}{l}\mathrm{I} / \mathrm{M} \\
\mathrm{no} .= \\
41\end{array}$ & $\begin{array}{l}\text { Ceft } \\
\text { no. }=75\end{array}$ & $\begin{array}{l}S \\
\text { no. }=20\end{array}$ & $\begin{array}{l}\mathrm{T} \\
\text { no. }=67\end{array}$ & $\begin{array}{l}\text { C } \\
\text { no. }= \\
69\end{array}$ \\
\hline \multirow{2}{*}{$\begin{array}{l}\text { Acinetobacter } \\
\text { (17) }\end{array}$} & $\mathbf{R}$ & $\begin{array}{l}17 \\
(100.0)\end{array}$ & - & $\begin{array}{l}17 \\
(100.0)\end{array}$ & $\begin{array}{l}16 \\
(94.1)\end{array}$ & $\begin{array}{l}16 \\
(94.1)\end{array}$ & $\begin{array}{l}16 \\
(100.0)\end{array}$ & $\begin{array}{l}17 \\
(100.0)\end{array}$ & $\begin{array}{l}15 \\
(100.0)\end{array}$ & $\begin{array}{l}17 \\
(100.0)\end{array}$ & $\begin{array}{l}14 \\
(93.3)\end{array}$ \\
\hline & $\mathrm{S}$ & - & $\begin{array}{l}17 \\
(100.0)\end{array}$ & - & $\begin{array}{l}1 \\
(5.9)\end{array}$ & $\begin{array}{l}1 \\
(5.9)\end{array}$ & - & - & - & - & $\begin{array}{l}1 \\
(6.7)\end{array}$ \\
\hline \multirow{2}{*}{$\begin{array}{l}\text { E.Coli } \\
\text { (7) }\end{array}$} & $\mathbf{R}$ & $\begin{array}{l}2 \\
(28.6)\end{array}$ & - & - & $\begin{array}{l}2 \\
(28.6)\end{array}$ & - & $\begin{array}{l}4 \\
(100.0)\end{array}$ & $\begin{array}{l}1 \\
(20.0)\end{array}$ & - & - & $\begin{array}{l}1 \\
(25.0)\end{array}$ \\
\hline & S & $\begin{array}{l}5 \\
(71.4)\end{array}$ & - & - & $\begin{array}{l}5 \\
(71.4)\end{array}$ & $\begin{array}{l}2 \\
(100.0)\end{array}$ & - & $\begin{array}{l}4 \\
(80.0)\end{array}$ & - & $\begin{array}{l}3 \\
(100.0)\end{array}$ & $\begin{array}{l}3 \\
(75.0)\end{array}$ \\
\hline \multirow{2}{*}{$\begin{array}{l}\text { Kleb. } \\
\text { Pheumoniae } \\
\text { (12) }\end{array}$} & $\mathbf{R}$ & $\begin{array}{l}8 \\
(66.7)\end{array}$ & - & $\begin{array}{l}4 \\
(100.0)\end{array}$ & $\begin{array}{l}6 \\
(50.0)\end{array}$ & $\begin{array}{l}6 \\
(85.7)\end{array}$ & $\begin{array}{l}3 \\
(42.9)\end{array}$ & $\begin{array}{l}8 \\
(80.0)\end{array}$ & $\begin{array}{l}1 \\
(100.0)\end{array}$ & $\begin{array}{l}4 \\
(66.7)\end{array}$ & $\begin{array}{l}5 \\
(71.4)\end{array}$ \\
\hline & S & $\begin{array}{l}4 \\
(33.3)\end{array}$ & $\begin{array}{l}4 \\
(100.0)\end{array}$ & - & $\begin{array}{l}6 \\
(50.0)\end{array}$ & $\begin{array}{l}1 \\
(14.3)\end{array}$ & $\begin{array}{l}4 \\
(57.1)\end{array}$ & $\begin{array}{l}2 \\
(20.0)\end{array}$ & - & $\begin{array}{l}2 \\
(33.3)\end{array}$ & $\begin{array}{l}2 \\
(28.6)\end{array}$ \\
\hline \multirow{2}{*}{$\begin{array}{l}\text { Pseudomonas } \\
\text { (29) }\end{array}$} & $\mathbf{R}$ & $\begin{array}{l}19 \\
(63.3)\end{array}$ & - & - & $\begin{array}{l}7 \\
(24.1)\end{array}$ & $\begin{array}{l}2 \\
(100.0)\end{array}$ & $\begin{array}{l}8 \\
(66.7)\end{array}$ & $\begin{array}{l}18 \\
(60.0)\end{array}$ & $\begin{array}{l}3 \\
(75.0)\end{array}$ & $\begin{array}{l}10 \\
(35.7)\end{array}$ & $\begin{array}{l}10 \\
(33.3)\end{array}$ \\
\hline & S & $\begin{array}{l}11 \\
(36.7)\end{array}$ & $\begin{array}{l}6 \\
(100.0)\end{array}$ & $\begin{array}{l}1 \\
(100.0)\end{array}$ & $\begin{array}{l}22 \\
(75.9)\end{array}$ & - & $\begin{array}{l}4 \\
(33.3)\end{array}$ & $\begin{array}{l}12 \\
(40.0)\end{array}$ & $\begin{array}{l}1 \\
(25.0)\end{array}$ & $\begin{array}{l}18 \\
(64.3)\end{array}$ & $\begin{array}{l}20 \\
(66.7)\end{array}$ \\
\hline \multirow{2}{*}{ TOTAL } & $\mathbf{R}$ & $\begin{array}{l}59 \\
(68.6)\end{array}$ & - & $\begin{array}{l}21 \\
(91.3)\end{array}$ & $\begin{array}{l}31 \\
(36.9)\end{array}$ & $\begin{array}{l}30 \\
(85.7)\end{array}$ & $\begin{array}{l}27 \\
(65.9)\end{array}$ & $\begin{array}{l}57 \\
(76.0)\end{array}$ & $\begin{array}{l}19 \\
(95.0)\end{array}$ & $\begin{array}{l}33 \\
(49.3)\end{array}$ & $\begin{array}{l}32 \\
(46.4)\end{array}$ \\
\hline & S & $\begin{array}{l}27 \\
(31.4)\end{array}$ & $\begin{array}{l}27 \\
(100 .)\end{array}$ & $\begin{array}{l}2 \\
(8.7)\end{array}$ & $\begin{array}{l}53 \\
(63.1)\end{array}$ & $\begin{array}{l}5 \\
(14.3)\end{array}$ & $\begin{array}{l}14 \\
(34.1)\end{array}$ & $\begin{array}{l}18 \\
(24.0)\end{array}$ & $\begin{array}{l}1 \\
(5.0)\end{array}$ & $\begin{array}{l}34 \\
(50.7)\end{array}$ & $\begin{array}{l}37 \\
(53.6)\end{array}$ \\
\hline
\end{tabular}

Ce = Cefuroxime; Co = Colistin; P = Piperacillin; G = Gentamicin; AS = Ampicillin-Sulbactam; I = Imipenem;

$M=$ Meropenem; Ceft $=$ Ceftazidime; $\mathrm{S}=$ Sulperazone; $\mathrm{T}=$ Tazobactam $; \mathrm{C}=$ Ciprofloxacin

The finding is similar to other studies demonstrating multi-drug resistant Pseudomonas spp. ${ }^{17,18}$ Several studies have demonstrated association of blood transfusion with infection in open fracture with twice amount of relative risk. ${ }^{12,15}$ In this study, blood transfusion has statistically significant correlation with resistant microorganisms ( $p$ value < 0.001). Other studies reported similar findings involving Gram-negative bacilli. ${ }^{19,20}$

Choice of prophylactic antibiotic was found in this study to be statistically significant ( $p$ value $<0.001$ ) to the development of resistance. This is in agreement with other studies where initial exposure to antibiotics especially broad spectrum types has significant statistical association with resistance of microorganisms, where even cross resistance to other antimicrobials were established. ${ }^{17}$ In this study, majority of patients were on antibiotics for more than 10 days consisting of $77.3 \%$ of cases which were longer than established guidelines. Duration of antibiotics was found to be statistically significant ( $p$-value $<0.001$ ), similar to a study by Gouliouris et al. involving Enterococcus spp. ${ }^{21}$
In our study involving infected open fractures, most of the cases underwent initial debridement less than 12 hours from incident with $57.9 \%$ but fail to show any statistical significance. Several studies reported no association of infection rate to timing of debridement but did not address resistance of microorganisms. $^{22}$ Most of the patients were admitted for more than 10 days which consisted of 78.2\%. Duration of admission was found to have statistically significant relation of resistance of microorganisms ( $p$-value $<0.001$ ). This is supported by multiple studies which demonstrated development of resistance in longer duration of admission. ${ }^{17,18}$ This finding seems to suggest a hospital acquired source of infection and resistance development via patient to patient transmission.

With regards to species of microorganisms, this study demonstrated involvement of Gram-negative bacteria in $65.94 \%$ of cases compared to $26.81 \%$ Gram-positive. This result is in disagreement with several studies which reported predominant Grampositive microorganisms ${ }^{9,23}$ but is similar to several others. ${ }^{5}$ With relation to Gustilo Anderson fracture 
grading however, Gram-positive was found to be predominant in Grade I and II while Gram-negative was predominant as grade increases; $42.9 \%$ in grade II, $67.2 \%$ in grade IIIA, $76.5 \%$ in grade IIIB and $85.7 \%$ in grade IIIC. Gram-negative microorganism was also found to become predominant as ISS score increases and in cases involving ICU admission.

The bacteriology of this study demonstrated Pseudomonas was the predominant Gram-negative bacteria with $24 \%$ involvement which is similar to a study done locally followed by Acinetobacter species with $13.6 \%$ compared to $E$. coli as reported by others. ${ }^{5}$ Staphylococcus aureus remain the predominant Gram-positive bacteria with $15.2 \%$ followed by MRSA with $13.6 \%$ but no longer constitute the major causative microorganism as reported by several studies. ${ }^{7,23}$

\section{CONCLUSION}

As there are not many studies in orthopaedics which address the risk factors of infections by resistant microorganisms, the findings of this study could assist in the management of open fractures. More importantly, certain guidelines and antibiotic regimes might need to be fine-tuned to achieve optimal results in managing such patients. This will also prevent the emergence of new multi-drug resistant microorganisms which are already a worldwide public health problem.

\section{LIMITATION OF STUDY}

There were several limitations to this study. Some patients' data were incomplete due to loss of patients' medical records in the filing office or were transferred to a different medical centre where they had to be dropped from the study. The microbial culture and sensitivity done in this centre do not allow complete antibiotic sensitivity for all cases, therefore, not all microorganisms were tested to certain antibiotics. Furthermore, culture and sensitivity are not performed when 3 or more microorganisms are cultured thereby limiting the number of microorganisms tested for antimicrobial susceptibility.

\section{REFFERENCES}

1. Madhi SA, Petersen K, Madhi A, Khoosal M, Klugman KP. Increased disease burden and antibiotic resistance of bacteria causing severe community-acquired lower respiratory tract infections in human immunodeficiency virus type 1 -infected children. Clin Infect Dis. 2000;31(1):170-6.

2. Bugler KE, Clement ND, Duckworth AD, et al. The epidemiology of open fractures in adults. A 15-year review. Injury. 2012;43(6):891-7.

3. Jabatan Keselamatan Jalanraya. 2014. Pelan Keselamatan Jalanraya Malaysia 2014-2020. Jabatan Keselamatan Jalanraya(JKJR), Kementerian Pengangkutan Malaysia

4. Ministry of Health M. National Antibiotic Guidelines 2014.; 2014.

5. Yusof NM, Khalid KA, Zulkifly AH, et al. Factors associated with the outcome of open tibial fractures. Malaysian J Med Sci MJMS. 2013;20 (5):47.

6. Chua W, Murphy D, Siow W, Kagda F, Thambiah J. Epidemiological analysis of outcomes in 323 open tibial diaphyseal fractures: a nine-year experience. Singapore Med J. 2012;53(6):385-9.

7. Mama M, Abdissa A, Sewunet T. Antimicrobial susceptibility pattern of bacterial isolates from wound infection and their sensitivity to alternative topical agents at Jimma University. Ann Clin Microbiol Antimicrob. 2014;13(1):14-23.

8. Wolfe CM, Cohen B, Larson E. Prevalence and risk factors for antibiotic-resistant communityassociated bloodstream infections. J Infect Public Health. 2014;7(3):224-32.

9. Naeemullah HS, Khan AH, Gul H, Baz KA. Common Organisms and their Sensitivity, in open fractures of the Extremities. Pak J Surg. 2012;28(3):186-92.

10. Davis SL, Perri MB, Donabedian SM, et al. Epidemiology and outcomes of communityassociated methicillin-resistant Staphylococcus aureus infection. J Clin Microbiol. 2007;45 (6):1705-11.

11. Dellinger EP, Caplan ES, Weaver LD, et al. Duration of Preventive Antibiotic Administration for Open Extremity Fractures. Arch Surg. 1988;123(3):333-9.

12. Bowen TR, Widmaier JC. Host classification predicts infection after open fracture. Clin Orthop Relat Res. 2005;433:205-11.

13. Hull PD, Johnson SC, Stephen DJG, Kreder HJ, Jenkinson RJ. Delayed debridement of severe open fractures is associated with a higher rate of deep infection. Bone Jt J. 2014;96(3):379-84. 14. Li Z, Zhou Z, Li P, Zeng W, Qing H, Tang W. 
Retrospective study on multidrug-resistant bacterium infections after rigid internal fixation of mandibular fracture. J Oral Maxillofac Surg. 2016;74(4):770-7.

15. Dellinger EP, Miller SD, Wertz MJ, Grypma M, Droppert $B$, Anderson PA. Risk of infection after open fracture of the arm or leg. Arch Surg. 1988;123(11):1320-7.

16. Caricato A, Montini L, Bello G, et al. Risk factors and outcome of Acinetobacter baumanii infection in severe trauma patients. Intensive Care Med. 2009;35(11):1964-9.

17. Harris $A D$, Perencevich $E$, Roghmann $M-C$, Morris G, Kaye KS, Johnson JA. Risk factors for piperacillin-tazobactam-resistant Pseudomonas aeruginosa among hospitalized patients. Antimicrob Agents Chemother. 2002;46(3):8548.

18. Aloush $V$, Navon-Venezia $S$, Seigman-Igra $Y$, Cabili S, Carmeli Y. Multidrug-resistant Pseudomonas aeruginosa: risk factors and clinical impact. Antimicrob Agents Chemother. 2006;50(1):43-8.

19. Gudiol C, Tubau F, Calatayud L, et al. Bacteraemia due to multidrug-resistant Gramnegative bacilli in cancer patients: risk factors, antibiotic therapy and outcomes. J Antimicrob Chemother. 2010;66(3):657-63.

20. Playford EG, Craig JC, Iredell JR. Carbapenemresistant Acinetobacter baumannii in intensive care unit patients: risk factors for acquisition, infection and their consequences. J Hosp Infect. 2007;65(3):204-11.

21. Gouliouris T, Warne B, Cartwright EJ, et al. Duration of exposure to multiple antibiotics is associated with increased risk of VRE bacteraemia: a nested case-control study. Journal of Antimicrobial Chemotherapy. 2018;73(6):1692-9.

22. Harley BJ, Beaupre LA, Jones CA, Dulai SK, Weber DW. The effect of time to definitive treatment on the rate of nonunion and infection in open fractures. J Orthop Trauma. 2002;16(7):484-90.

23. Gustilo RB, Anderson JT. Prevention of infection in the treatment of one thousand and twenty-five open fractures of long bones: retrospective and prospective analyses. J Bone Joint Surg Am. 1976;58(4):453-458. 